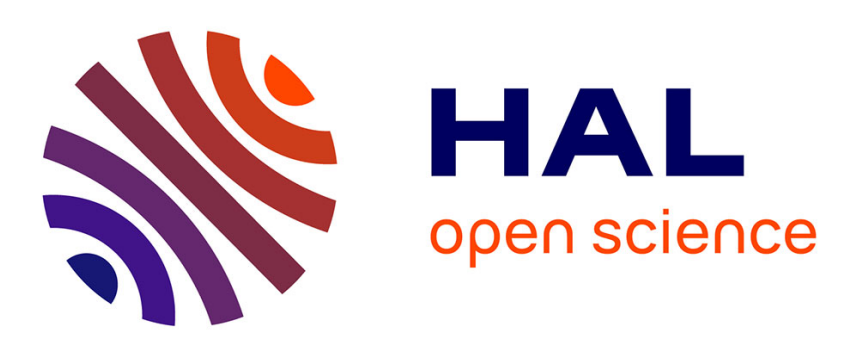

\title{
Intelligent distributed and supervised flow control methodology for production systems.
}

\author{
Karim Tamani, Reda Boukezzoula, Georges Habchi
}

\section{To cite this version:}

Karim Tamani, Reda Boukezzoula, Georges Habchi. Intelligent distributed and supervised flow control methodology for production systems.. Engineering Applications of Artificial Intelligence, 2009, 22, pp. 1104-1116. 10.1016/j.engappai.2009.01.007 . hal-00403801

\section{HAL Id: hal-00403801 \\ https://hal.univ-smb.fr/hal-00403801}

Submitted on 8 Jan 2013

HAL is a multi-disciplinary open access archive for the deposit and dissemination of scientific research documents, whether they are published or not. The documents may come from teaching and research institutions in France or abroad, or from public or private research centers.
L'archive ouverte pluridisciplinaire HAL, est destinée au dépôt et à la diffusion de documents scientifiques de niveau recherche, publiés ou non, émanant des établissements d'enseignement et de recherche français ou étrangers, des laboratoires publics ou privés. 


\title{
Intelligent distributed and supervised flow control methodology for production systems
}

\author{
Karim Tamani $^{1}$, Reda Boukezzoula ${ }^{1}$, Georges Habchi ${ }^{2}$ \\ ${ }^{1}$ University of Savoie, Polytech'Savoie, ${ }^{1}$ LISTIC Laboratory, \\ ${ }^{2}$ SYMME Laboratory, \\ Domaine Universitaire - BP 80439, 74944 Annecy-le-Vieux Cedex France
}

\{karim.tamani, reda.boukezzoula, georges.habchi\}@univ-savoie.fr

\begin{abstract}
This paper deals with the development of an intelligent distributed and supervised control approach for highvolume production systems in which the flow of parts can be approximated by a continuous (fluid) model. The proposed approach is based on the decomposition of the production system into elementary modules in order to reduce the control design computational complexity. In this context, a two levels control structure is proposed. At the local level, a surplus-based principle is adopted to regulate the production flow for each module according to the distributed structure. The proposed control methodology decides how to adjust the production rate in order to avoid system overloading and eliminate machine starvation or blocking. In this context, the local control law is synthesized by using the Takagi-Sugeno fuzzy systems. At the high level, a supervisory controller is designed to improve the overall system performances. A supervisor provides an additive component for each local controller when the overall system performances deviate from their acceptable domains (degraded mode). This is done by combining both local and global information into a unified formalism by using aggregation operators and according to fuzzy interval representation of the desired objectives. Finally, the feasibility of the proposed methodology is validated with simulation examples.
\end{abstract}

Key words: Production Systems; Intelligent Control; Distributed Fuzzy Control; Supervisory Control; Aggregation Operators; Continuous-Flow Simulation.

\section{Introduction}

Many industrial production systems, such as semiconductor manufacturing, generate typical processes of largescale, time-varying and stochastic systems. They involve different kinds of operation (transformation, assembly or disassembly), operate in an uncertain and unpredictable environment and manufacture a high-volume and medium-variety of products (product types generally are limited). These features make the system complex. Generally, these processes are designed to operate continuously under some optimal operating conditions. However, the operations may deviate away from the normal operating modes due to changing of the production demands properties or the environmental conditions. Therefore, a control decision strategy should be designed so as the most important performance measures (throughput, backlog, work-in-process, etc.) stay within the desired specifications.

Actually, complexity and uncertainty seriously limit the effectiveness of the conventional modelling and control approaches. As a result, complex production systems are not amenable to accurate and exact modelling. In this framework, in order to overcome the system complexity analysis and its control design, a decomposition of the system into elementary subsystems is frequently considered $[42,43,46]$. Thus, a production system composed of $N$ machines and a set of buffers can be viewed as a collection of a set of $N$ elementary production modules $P M(i), i=1, \ldots, N$. Each one is defined by a machine and its sets of upstream and downstream buffers. In this context, the study of the global system can be handled from a generic analysis of an elementary subsystem. According to this decomposition, a distributed control structure can be adopted to avoid the inflexibility and the rigidity, conventionally associated with the centralized control architecture. Indeed, it is now recognized that distributed control architectures have a number of inherent advantages, such as modularity, reconfigurability, adaptability, fault tolerance, extensibility, etc [14, 45].

Since an exact analytical control design cannot be determined in realistic manufacturing conditions, intelligent methods seem to be a very effective tool to develop control strategies for these systems where complete mathematical models are not available. Indeed, intelligent control offers an alternative to conventional control for designing controllers. In this case, the structure and the consequent outputs in response to external 
disturbances and environmental conditions are determined by empirical evidence, i.e., observed input/output behaviour of the system.

In the literature, the intelligent distributed control for production systems has been introduced either through some paradigms such as holonic systems [6, 22, 23, 26] and agent-based systems [36, 37] or by using artificial intelligent techniques such as neural networks and fuzzy logic [1,33, 46]. In the former case, the distributed control means that the control algorithm is distributed over a number of entities (software components) that combine their calculation power and their local knowledge to optimise the global performances. In the latter case, from a control theory point of view, the distributed control methodology consists in performing a controller for each production module independently to preserve the simplicity and computational advantages. In this article, the proposed approach is based on the last idea where a fuzzy control using a Takagi-Sugeno fuzzy system [40] is adopted. The application of fuzzy techniques in distributed control structures is not new. Indeed, several works have been already published. For example, in [33] the authors propose a distributed neuralnetwork scheduling methodology approaching machine scheduling as a control regulation. In $[1,12]$, complex control problems of scheduling and planning have been addressed in a distributed fashion by using fuzzy control theory. In [46], Tsourveloudis et al. develop a distributed fuzzy system for work-in-process inventory control of unreliable machines.

It is well-known that if distributed control architectures offer prospects of reduced complexity, flexibility and robustness against disturbances, they cannot guarantee optimal performances and the system behaviour can be unpredictable [6]. Usually, the elements of these architectures have no access to global information and, therefore, global performances cannot be guaranteed. Moreover, the presence of multiple objectives which are often conflicting, such as short lead time, timely delivery, low WIP, etc., have compelled researchers to look for more appropriate control methods [25]. In this context, in order to improve the global performances, distributedsupervised control structures have emerged as an interesting and powerful solution.

The role of a supervisor consists in elaborating the production plans for the entities under its coordination domain by using performance indicators characterizing the overall system current behaviours [6]. In this case, the supervisor provides an action able to tune the distributed controllers to ultimately achieve desired specifications [31]. For example, in order to improve the WIP performance, the authors [20] introduce a fuzzy supervisor to tune the lower level of the distributed fuzzy controllers given in [46]. In [47], an evolutionary algorithm strategy is used for the optimisation of the approaches provided in [46] and [20]. In [48] a model based predictive supervisory controller for semiconductor re-entrant lines is developed. However, in the most proposed approaches, the dynamic of the internal production modules and the influence of the conflicting objectives in the supervisor synthesis are not explicitly taken into account. The method proposed in this article aims at resolving these problems where a two-level structure (a supervisor at the higher level and a distributed fuzzy controllers at the lower level) is developed. Thus, the control architecture is characterized by:

- Distributed local and autonomous controllers based on local information. Indeed, each control module is designed in order to regulate the production-flow at each production module by adjusting the machine processing rates with regard to its maximum production capacity.

- A supervisory controller able to combine the overall performance measures such as a best compromise is achieved between the multiple and possibly conflicting objectives. Indeed, when the global performances deviate from their acceptable domains (objectives), the supervisor provides an additive component which either distributes the remaining production capacity or reduces the production throughput. This is done according to the actual processing rate of each production module given by the local control law. The proposed supervisory decision-making process is based on the aggregation operators and fuzzy interval representation of the objectives.

To analyze the proposed method and its performances, continuous-flow simulation techniques based on fluid modelling are adopted. In this case, in contrast with discrete modelling, it is not necessary to track individual parts in the model evaluation. Indeed, the part movement can be approximated by a continuous-flow (fluid) so that the dimension of the model is reduced $[4,5,17,24,35]$.

The rest of the article is organized as follows. Section 2 introduces the problem statements and faces the major questions considered in this article. Section 3 develops a distributed fuzzy control methodology. Section 4 describes the aggregation mechanisms of the supervised approach. Section 5 includes an extension of the proposed control methodology to the multiple-part-type systems and simulation results along with comparisons between the unsupervised distributed fuzzy control and the supervised one. Our contributions are summarized in Section 6. 


\section{Problem statements and assumptions}

A production system is usually viewed as a network of machines and buffers. In this context, when considering a production system composed of $N$ machines and according to the operation type (transformation, assembly and disassembly), it may be decomposed into $N$ basic production modules $P M(i)$. Each one is composed of a machine $M_{i}$ and its sets of upstream and downstream buffers denoted respectively by $B_{+}(i)$ and $B_{-}(i)$. For instance, in the case of a transformation line illustrated in Fig. 1, the production module is defined as $P M(i)=$ $\left\{B_{i-1}, M_{i}, B_{i}\right\}$.

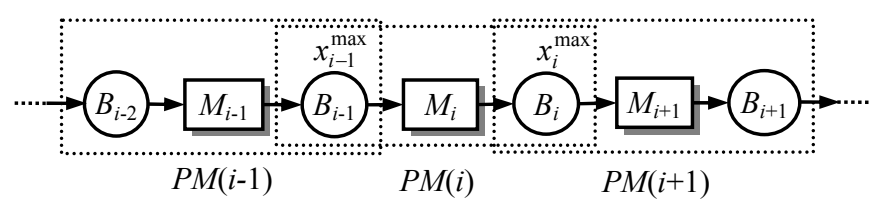

Fig. 1. Transformation line.

For the sake of simplicity, the developments are given for a single-part-type transformation line. The generalization to the multiple-part-type production systems is detailed in paragraph 5.2.1. The control design when assembly and/or disassembly operations are involved is discussed at the end of section 3 . In the sequel, the following assumptions are assumed:

- The machines are unreliable, where their uptimes and downtimes are assumed to be exponentially distributed with rates $\lambda_{i}$ and $\mu_{i}$ respectively.

- The intrinsic availability of $M_{i}$ (when taken in isolation) is given by $e_{i}=\lambda_{i} /\left(\lambda_{i}+\mu_{i}\right)$.

- $u_{i}^{\max }=1 / \tau_{i}$ is the maximum production rate at which $M_{i}$ can operate with a processing time $\tau_{i}$.

- The demand is constant with a rate $d$.

- The condition of feasibility of the demand rate is given as follows [32]:

$$
d \leq \min _{i=1, \ldots, N}\left\{d_{i}^{\max }\right\} \text {, with } d_{i}^{\max }=e_{i} u_{i}^{\max }
$$

- $u_{i}(t)$ is the instantaneous production rate of an operation performed on $M_{i}$ and $\alpha_{i}(t)$ is the machine state $\left(\alpha_{i}(t)=1\right.$ if $M_{i}$ is up and $\alpha_{i}(t)=0$ if it is down). Then,

$$
\begin{aligned}
\text { if } \alpha_{i}(t) & =0, \quad u_{i}(t)=0 \\
\text { if } \alpha_{i}(t) & =1, \quad 0 \leq u_{i}(t) \leq u_{i}^{\max }
\end{aligned}
$$

- $r_{i}(t)$ is the fraction of the capacity of $M_{i}$ devoted for processing at time $t$ (the weighting factor of the maximum production rate). It represents the control variable which fix the production rate and given by:

$$
r_{i}(t)=\frac{u_{i}(t)}{u_{i}^{\max }}, \text { with } r_{i}(t) \in[0,1]
$$

- $x_{i}^{\text {max }}$ is the maximal capacity (size) of the buffer $B_{i}$.

- $x_{i}(t)$ is the level of $B_{i}$ at time $t$. Its dynamic is given by (Fig. 1):

$$
\dot{x}_{i}(t)=u_{i}(t)-u_{i+1}(t) \text {, with } 0 \leq x_{i}(t) \leq x_{i}^{\max }
$$

This dynamic equation represents the basis of the continuous model used in simulation. Indeed, when considering a production system composed of $N$ production modules, its dynamic is governed by $N$ differential equations in the form (4).

- The system surplus dynamic which defines the cumulative difference between production and demand is defined as:

$$
\dot{s}_{i}(t)=u_{i}(t)-d
$$

When $s_{i}(t)$ is positive, an inventory surplus is observed and when it is negative, a backlog is occurred.

Giving a set of production modules constituting the production system, the originality of this work lies in answering given to the following questions:

- What is the best control structure able to preserve the simplicity and the computational advantages? 
- For a feasible demand rate, is it possible to synthesize a tracking policy able to maintain a bounded surplus for each production module?

- Giving tolerance domains defining normal operating conditions and specified by the designer for the overall objectives, is it possible to synthesize a control strategy able to maintain the system performances within these tolerance domains?

- How the local and global information are aggregated to achieve the desired performances?

\section{Distributed Fuzzy Control Architecture}

Let us consider a general production system decomposed in $N$ production modules $P M(i)$. Each one is associated to a fuzzy controller $F C(i)$ resulting on a distributed fuzzy control architecture as illustrated in Fig. 2. The major advantage of this architecture resides in its modularity and distributivity that enhance the flexibility and make easy its implementation for complex production systems.

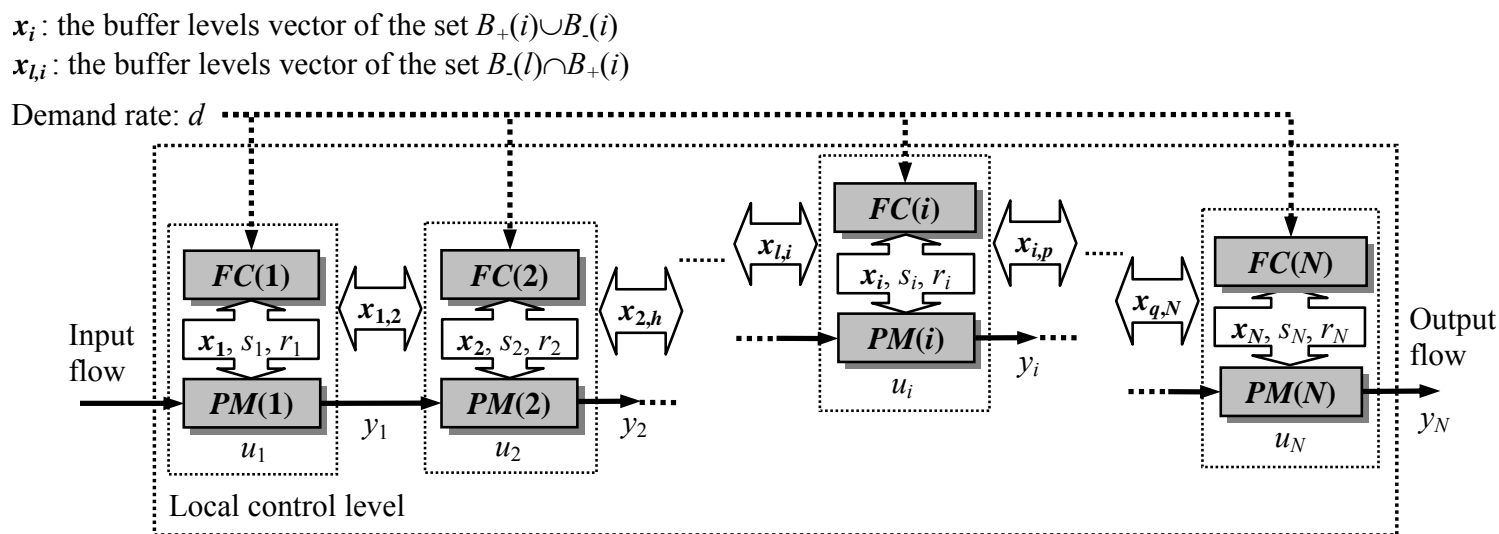

Fig. 2. Distributed fuzzy control architecture.

In the sequel, the control synthesis is handled through a generic analysis and design of one fuzzy controller $F C(i)$ for a transformation operation (Fig. 1). In this case, the control structure is illustrated in Fig. 3.

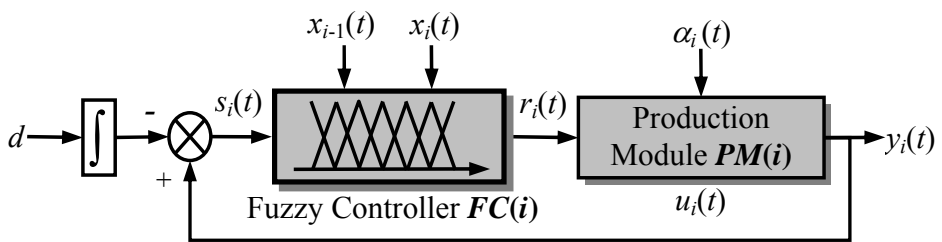

Fig. 3. Controlled production module.

Thus, giving a production module $P M(i)$ and a feasible demand rate $d$, a fuzzy controller $F C(i)$ is synthesised with the objectives of:

- Tracking the demand trajectory by reducing the difference between the cumulative production and demand (the surplus),

- Synchronising the operations by avoiding overload and eliminating machine starvation or blocking,

In this case, a controller attempts to keep the buffers neither full nor empty by regulating the machine production rate. The following input variables are then considered:

- The levels of upstream and downstream buffers $x_{i-1}(t)$ and $x_{i}(t)$.

- The production surplus $s_{i}(t)$ of $P M(i)$.

The output variable of the controller is the weighting factor $r_{i}(t)$ adjusting the production rate of $P M(i)$ between zero and its maximum. According to a fuzzy control vision, this is achieved according to the two following rules:

- If the surplus level is satisfying, then try to prevent starving or blocking by increasing or decreasing the production rate of the machine.

- If the surplus is either too low or too high, then produce respectively with the maximum or zero rate.

Moreover, the expert distinguishes several zones to be controlled by observing the error between the production and the desired demand (surplus and backlog). According to expert observations and analysis, a partitioning of 
the universes of discourse in fuzzy subsets is given. This partitioning consists to affect labels allowing the description of the numerical values using words. Indeed, the expert interprets the preceding situations by linguistic terms according to whether the actual production is "in delay", "correct" or "in advance" with regard to the demand. This classification is easily retranscribed in terms of "Low", "Normal" and "High" by defining the surplus as the difference between machine production and the demand. The "normal" level of surplus represents a hedging level [3] able to absorb the disturbances due to the machine failures.

The expert is also sensitive to the events that may occur, especially the changes in buffer levels according to the machine states. In this case, the buffers may be "Empty", "Almost Empty", "Normal", "Almost Full" or "Full". The partitioning of different universes of discourse is illustrated in Fig. 4.

Lastly, according to his perception, the expert determines the action to be adopted concerning the production rate: "Zero", "Small", "Average", "Large" or "Maximum". These terms can be interpreted with regard to the maximum production rate $u_{i}^{\max }$ by constant values giving the fraction of capacity devoted to processing: $0,0.25$, $0.5,0.75$ or 1 .

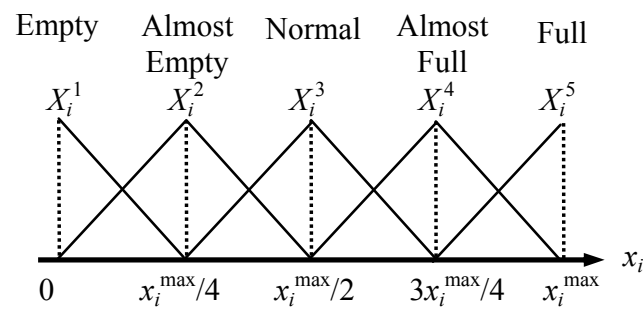

(a)

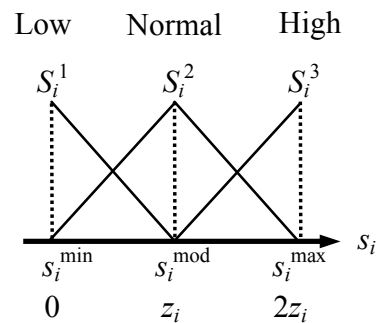

(b)

Fig. 4. Fuzzy partitions associated with the variables: (a) buffer level, (b) surplus.

It appears from this partitioning that the determination of the buffer sizes and the hedging level are crucial for the controller performance. These parameters are estimated by the heuristic described in [3]. This heuristic is based on the formulation of the relationship among starvation, blockage, demand and the parameters of the machines. In the obtained relation, the buffers sizes $x_{i}^{\max }$ are approximated by a nonlinear optimization problem minimizing the buffer hedging level $z_{i}^{s}$ and space $z_{i}^{b}$, which represent respectively the buffer level and the available free space in the buffer when the production module reaches its hedging point. The hedging point $z_{i}$, which is used to fix the surplus universe of discourse, is estimated from the average surplus loss caused by failure, starvation and blockage, such that the final product inventory and backlog are minimized. The detailed derivation of this heuristic is given in $[2,3]$.

According to the universe of discourse partitioning, the fuzzy controller $F C(i)$ can be formalized as a TakagiSugeno fuzzy system [40] given by a collection of rules in the following form:

$$
R_{i}^{\left(i_{1}, i_{2}, i_{2}\right)} \quad: \quad \text { IF } x_{i-1} \text { is } X_{i-1}^{i_{1}} \text { and } x_{i} \text { is } X_{i}^{i_{2}} \text { and } s_{i} \text { is } S_{i}^{i_{3}} \text {, then } r_{i}=\varphi_{i}^{\left(i_{1}, i_{2}, i_{3}\right)}
$$

where:

- $x_{i-1}$ and $x_{i}$ are respectively the levels of the upstream and downstream buffers of $M_{i}$ and $s_{i}$ the production surplus.

- $\quad X_{i-1}^{i_{1}}, X_{i}^{i_{2}}$ and $S_{i}^{i_{3}}$ correspond respectively to the $i_{k}$ th linguistic term of the input variable $x_{i-1}, x_{i}$ and $s_{i}$ (Fig. 4).

- $\varphi_{i}^{\left(i_{1}, i_{2}, i_{3}\right)}$ is the real value involved in the rule conclusion indexed by $\left(i_{1}, i_{2}, i_{3}\right)$.

For the input variables and when assuming a strict partitioning of the different universe of discourse, the output generated by the fuzzy controller is given by:

$$
r_{i}(t)= \begin{cases}0 & \text { if } \alpha_{i}(t)=0 \\ \sum_{\left(i_{1}, i_{2}, i_{3}\right) \in I} \xi_{i}^{\left(i_{1}, i_{2}, i_{3}\right)}\left(x_{i-1}, x_{i}, s_{i}\right) \cdot \varphi_{i}^{\left(i_{1}, i_{2}, i_{3}\right)} & \text {,if } \alpha_{i}(t)=1\end{cases}
$$

where $\xi_{i}^{\left(i_{1}, i_{2}, i_{3}\right)}\left(x_{i-1}, x_{i}, s_{i}\right)=\mu_{X_{i-1}^{i_{1}}}\left(x_{i-1}\right) \cdot \mu_{X_{i}^{i_{2}}}\left(x_{i}\right) \cdot \mu_{S_{i}^{i_{i}}}\left(s_{i}\right)$ represents the truth value of the premises of the rules (6), and $I=I_{1} \times I_{2} \times I_{3}$ indicates the set of labels representing the rules base, with $i_{v} \in I_{v}=\{1, \ldots, 5\}$ for $v=1,2$ and $i_{3} \in I_{3}=\{1,2,3\}$. Thus, the complete base of rules for a fuzzy controller of a transformation module is 
composed of 75 rules [43]. The different output values of the rule conclusions are obtained from expertise and summarized in Table 1.

Table 1. The rules base of $F C(i)$ for a transformation module.

\begin{tabular}{|c|c|c|c|c|}
\hline \multirow[t]{2}{*}{ Rule } & \multicolumn{3}{|l|}{ Inputs } & \multirow{2}{*}{$\frac{\text { Output }}{\varphi_{i}^{\left(i_{1}, i_{2}, i_{3}\right)}}$} \\
\hline & $X_{i-1}^{i_{1}}$ & $X_{i}^{i_{2}}$ & $S_{i}^{i_{3}}$ & \\
\hline 1 & Empty & ANY & ANY & 0 \\
\hline 2 & ANY & Full & ANY & 0 \\
\hline 3 & Not Empty & Not Full & Low & 1 \\
\hline 4 & Almost Empty & Not Full & High & 0 \\
\hline 5 & Normal & Not Full & High & 0 \\
\hline 6 & Almost Full & Empty & High & 0.75 \\
\hline 7 & Almost Full & Almost Empty & High & 0.5 \\
\hline 8 & Almost Full & Normal & High & 0.25 \\
\hline 9 & Almost Full & Almost Full & High & 0.25 \\
\hline 10 & Full & Empty & High & 1 \\
\hline 11 & Full & Almost Empty & High & 0.75 \\
\hline 12 & Full & Normal & High & 0.5 \\
\hline 13 & Full & Almost Full & High & 0.25 \\
\hline 14 & Almost Empty & Empty & Normal & 0.5 \\
\hline 15 & Almost Empty & Almost Empty & Normal & 0.25 \\
\hline 16 & Almost Empty & Normal & Normal & 0.25 \\
\hline 17 & Almost Empty & Almost Full & Normal & 0.25 \\
\hline 18 & Normal & Empty & Normal & 1 \\
\hline 19 & Normal & Almost Empty & Normal & 0.75 \\
\hline 20 & Normal & Normal & Normal & 0.5 \\
\hline 21 & Normal & Almost Full & Normal & 0.25 \\
\hline 22 & Almost Full & Empty & Normal & 1 \\
\hline 23 & Almost Full & Almost Empty & Normal & 0.75 \\
\hline 24 & Almost Full & Normal & Normal & 0.5 \\
\hline 25 & Almost Full & Almost Full & Normal & 0.25 \\
\hline 26 & Full & Empty & Normal & 1 \\
\hline 27 & Full & Almost Empty & Normal & 1 \\
\hline 28 & Full & Normal & Normal & 1 \\
\hline 29 & Full & Almost Full & Normal & 0.5 \\
\hline
\end{tabular}

ANY means that the corresponding input variable can be substituted by any linguistic value of its set of terms, while Not $X$ means all the terms except $X$.

Some remarks and design considerations can now be expressed concerning the control methodology presented above:

- It is important to note that most applications using fuzzy controllers $[1,12,20,34,38,46]$ are based on fuzzy Mamdani systems [27] where the nonlinear min/max operators are used in the inference mechanism. In the context of our research, we did not find any advantage to exploit the min/max operators at least in a control context. On the contrary, the use of the latter operators involves the appearance of nonlinearities which may potentially deteriorate the control performances and the stability. Conversely, it has been shown in $[7,16]$ that the Takagi-Sugeno systems are more suitable in a local control design strategy.

- The TS fuzzy system present structural properties [39] that allow exact piecewise multi-linear representation. This representation permit to integrate the fuzzy control design in some adaptive or learning strategies to specify the rule base parameters. In our previous work, adaptive fuzzy control strategy has been proposed to adjust some parameters so that the tacking error (surplus) converges to zero [41]. However, the adaptive strategy is time consuming and, in some cases, induces instability problems. This is the reason for which the expertise is used to select the rule base conclusions in this article.

- In the case of assembly and disassembly modules, the proposed methodology can be extended by tacking into account more than one upstream buffer level (assembly module) or more than one downstream buffer level (disassembly module) as input variables in the fuzzy controller synthesis. The rule bases are then built in such a way to synchronize the operations with regards to the surplus and buffer levels. In this case, the applicability of the distributed fuzzy control structure has been shown in [42, 43] by considering a test case of a multiple-product reentrant production system involving assembly and disassembly operations. However, the augmentation of the input-output variables can induce difficulties in the control synthesis (rule base conception and synchronization). In $[42,43]$, the assembly and disassembly modules have been limited to two upstream buffers and two downstream buffers respectively. 


\section{Distributed-supervised Fuzzy Control Architecture}

Although the distributed structure can give sometimes good control performances [14, 45, 46], it does not guarantee optimal control performances since the global information is not integrated in the distributed local control synthesis (performances are considered myopically). Indeed, the production objectives which are often conflicting are evaluated by global performance indicators. Therefore, an aggregation methodology between different objectives has to be sought. In order to achieve this goal, higher supervisor based on global performance indicators aggregation is proposed.

In the literature related to control-systems, a supervisor is viewed as a controller that uses global information to adjust the current behaviour of the overall system when a degraded operating mode is detected. Potentially, this can be achieved either by reconfiguring and tuning a new local control law or by providing an additive component to the local controller. In both cases, the supervisory action aims at maintaining the overall system in the normal operating mode and relieves decision-making process in a degraded situation to ultimately achieve desired specifications (objectives) [31]. The approach adopted in this paper is based on the second principle where the supervisor is synthesized without modifying the proposed distributed local architecture. The supervisor takes into account the state of each production module and is able to cope with conflicting objectives.

The proposed distributed-supervised control structure is illustrated in Fig. 5. Starting from a set of performance indicators $\left\{P_{1}, \ldots, P_{L}\right\}$ associated to the objectives $\left\{P_{1}{ }^{\text {obj }}, \ldots, P_{L}{ }^{\text {obj }}\right\}$ that define the tolerance domains of these indicators, the supervisory controller aims at determining an additive component $r_{s_{i}}$ to the local control law $r_{c_{i}}$ in order to improve the performances when a degradation is appeared. In this case, the production rate in relation with the global control law can be written as:

$$
u_{i}(t)=\left(r_{c_{i}}(t)+r_{s_{i}}(t)\right) \cdot u_{i}^{\max }=r_{i}(t) u_{i}^{\max }
$$

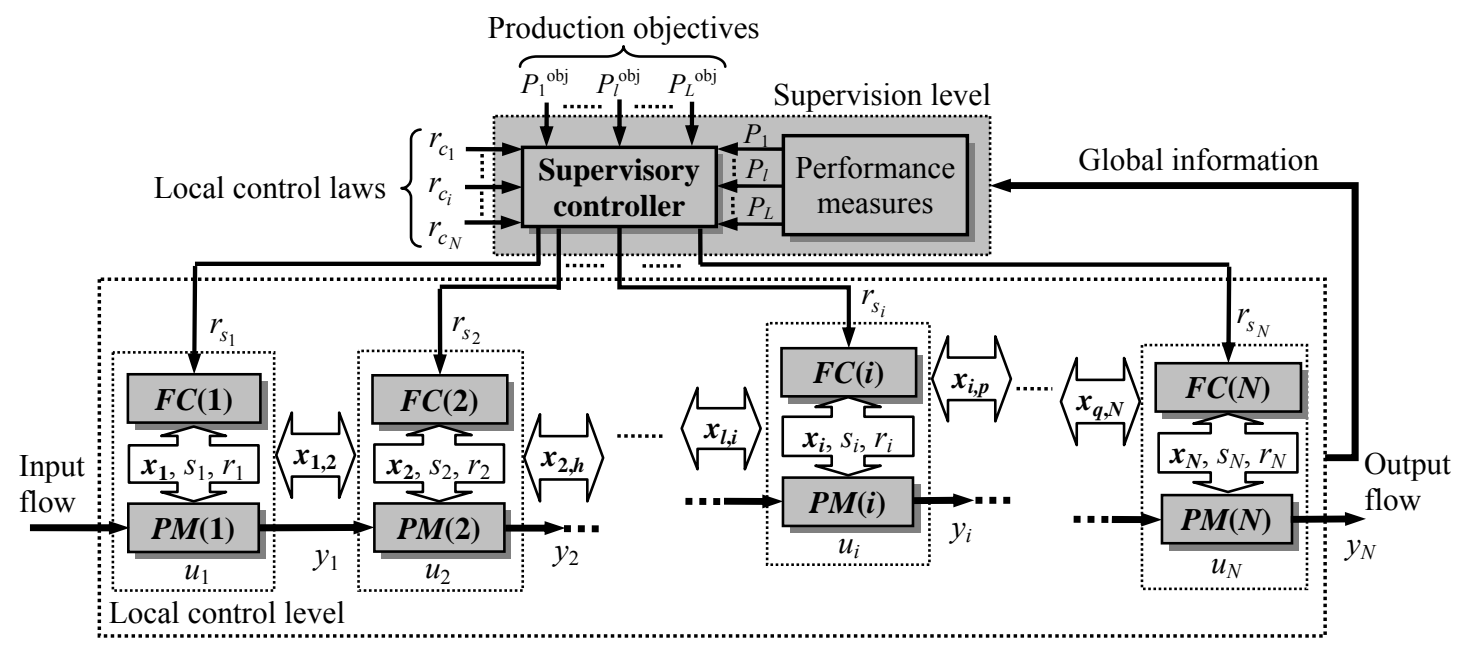

Fig. 5. Supervisory control architecture.

The supervision mechanism reported in this paper expands and improves our precedent work given in [42, 43]. The resulted supervisory action is deployed by either allocating the remaining capacity or reducing the production rate. This action is resulted from the aggregation of both local and global information to supervise the production flow. The key idea of the proposed method resides in the fuzzy intervals representation of the objectives and an aggregation mechanism based on mathematical operators.

\subsection{Fuzzy intervals representation of the objectives}

It is well know that fuzzy interval representation of the objectives permits preference degree specifications. Indeed, a fuzzy performance objective can be considered as the fuzzy quantity that should be reached. For example, when one wants to reach a performance for the WIP one would rather specify it by a fuzzy expression like "about 5 products", and model it by a membership function rather than give precise information. Based on expertise, this approach consists in associating a membership function $\mu$ with the performance indicator. Many shapes can be used such as triangular, trapezoidal or Gaussian. In this case, the grade of membership can be considered as a degree of preference. The higher degree is the more preferred value to be reached by the performance indicator is. The $\alpha$-cut of this fuzzy objective is the set of all the values satisfying the performance at least with a preference degree of $\alpha$ and the support is the set of values with preference greater than 0 . The 
kernel is the subset of the most preferred values. The complement of the support is the set of undesirable values. Thus, fuzzy intervals are interesting tools for characterizing result reliability and developing sensitiveness studies. Moreover, fuzzy intervals encapsulate within a single formalism the optimistic case (total satisfaction) and the pessimistic one (fully unsatisfied).

Giving a trapezoidal fuzzy interval $P_{l}^{\text {obj }}$ that represents the objective associated to the performance indicator $P_{l}$ as illustrated in Fig. 6, the following notations are used:

- The support and kernel are respectively denoted:

$$
S\left(P_{l}^{\mathrm{obj}}\right)=P_{l}^{\mathrm{obj}}(0)=\left[\left(P_{l}^{\mathrm{obj}}\right)^{-}(0),\left(P_{l}^{\mathrm{obj}}\right)^{+}(0)\right] \text { and } K\left(P_{l}^{\mathrm{obj}}\right)=P_{l}^{\mathrm{obj}}(1)=\left[\left(P_{l}^{\mathrm{obj}}\right)^{-}(1),\left(P_{l}^{\mathrm{obj}}\right)^{+}(1)\right]
$$

- In order to specify the fuzzy interval shape, two additional functions are used to link the support and the kernel values according to the vertical dimension. These functions, denoted by $\left(P_{l}^{\text {obj }}\right)^{-}$(the increasing part in Fig. 6) and $\left(P_{l}^{\mathrm{obj}}\right)^{+}$(the decreasing part in Fig. 6), are respectively called the left and right profiles [9, 10, 13]. They are defined by (9) where $\alpha$ is given on the vertical dimension (the $\alpha$-cut, $\alpha \in[0,1]$ ).

$$
\left\{\begin{array}{l}
\left(P_{l}^{\mathrm{obj}}\right)^{-}(\alpha)=\inf \left\{P_{l} \mid \mu_{P_{l}^{\mathrm{obj}}}\left(P_{l}\right) \geq \alpha ; P_{l} \geq\left(P_{l}^{\mathrm{obj}}\right)^{-}(0)\right\}=(1-\alpha) \cdot\left(P_{l}^{\mathrm{obj}}\right)^{-}(0)+\alpha \cdot\left(P_{l}^{\mathrm{obj}}\right)^{-}(1) \\
\left(P_{l}^{\mathrm{obj}}\right)^{+}(\alpha)=\sup \left\{P_{l} \mid \mu_{P_{l}^{\mathrm{obj}}}\left(P_{l}\right) \geq \alpha ; P_{l} \leq\left(P_{l}^{\mathrm{obj}}\right)^{+}(0)\right\}=(1-\alpha) \cdot\left(P_{l}^{\mathrm{obj}}\right)^{+}(0)+\alpha \cdot\left(P_{l}^{\mathrm{obj}}\right)^{+}(1)
\end{array}\right.
$$

- Any $\alpha$-cut of a fuzzy performance is the set of the values satisfying the objective $P_{l}^{\text {obj }}$ at least with a degree $\alpha[8]$. Thus, the switching between the different operating modes is conducted in a gradual way by the left and right profiles of the fuzzy interval.

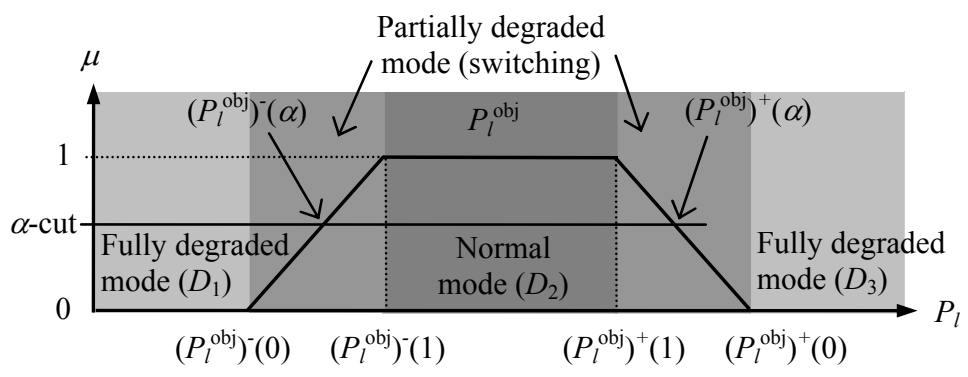

Fig. 6. Trapezoidal fuzzy interval representation.

The satisfaction may be total when the performance indicator evolves within the kernel (normal mode), not satisfactory at all if it is outside the support (fully degraded mode), and not satisfactory with different degree when it is bounded by the left or right profiles (partially degraded mode) (Fig. 6). These different situations distinguish the operating modes of the production system.

\subsection{Supervisory control based aggregation operators}

In the supervisory control design, two aggregation mechanisms are developed. The first one is based on the aggregation of the actions. Indeed, for each performance indicator, an appropriate action is determined independently and the resulted action of the supervisor is obtained by the aggregation of these various actions. The second mechanism consists on an aggregation of the performance indicator measures to determine the action associated with the aggregated objective.

The main difference between these two approaches resides in the application of the aggregation operator. Indeed, in the first mechanism, the aggregation is based on the assumption that all the actions have the same importance. This is due to the fact that each action is designed according to the satisfaction degree of its objective taken independently from the others. In this case, the objectives are considered without specifying any preference or any interaction between them. However, the objectives can present conflict behaviours resulted from interaction between the related performances. Thus, the second proposed mechanism is used in this situation where the aggregation is done according to the specified preferences and, if any, their interactions.

\subsubsection{The aggregation methodology of actions}

This methodology of supervision is designed according to the two following steps:

- For each performance indicator $P_{l}$, the associated action $r_{s_{i}}^{l}$ is determined according to the system operating mode. 
- The obtained actions $r_{s_{i}}^{l}$ for $l=1, \ldots, L$, are combined according to an aggregation operator providing the additive component $r_{s_{i}}$.

The principle of the methodology is illustrated in Fig. 7.

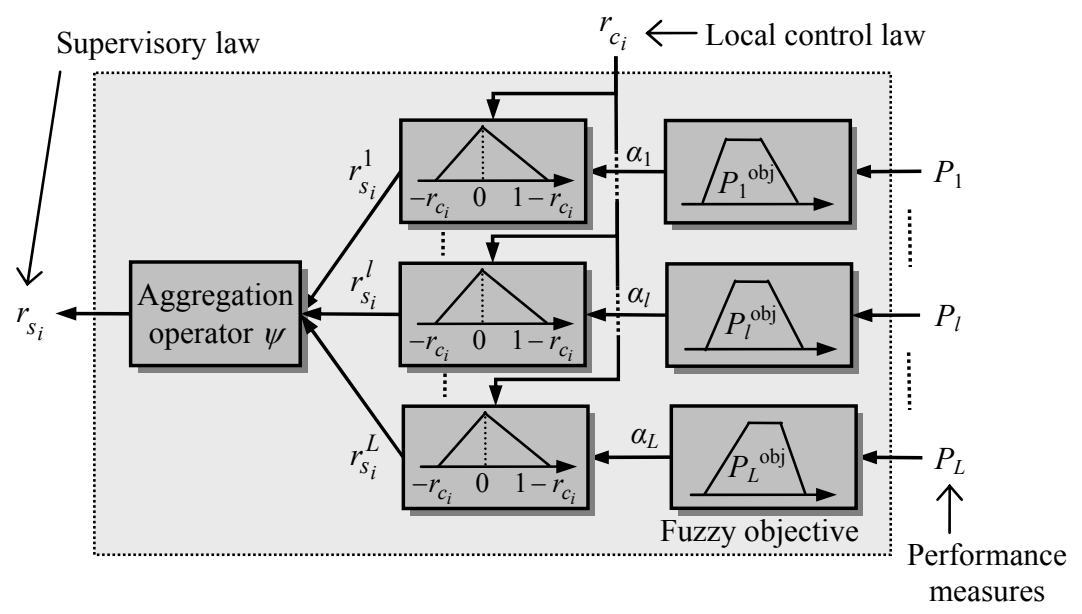

Fig. 7. Synopsis of the aggregation principle of actions.

Let us consider the objective $P_{l}^{\text {obj }}$ represented by its fuzzy interval as illustrated in Fig. 6. For a performance measure $P_{l}$, the associated action $r_{s_{i}}^{l}$ is determined according to the following statements:

- If $P_{l}$ evolves within the kernel of $P_{l}^{\text {obj }}$, the system behaviour is in normal mode (domain $D_{2}$ in Fig. 6). This means that the satisfaction degree of the objective is total $(\alpha=1)$. In this case, the supervisor does not provide additive component:

$$
\text { If } P_{l} \in P_{l}^{\text {obj }}(1) \text {, then } r_{s_{i}}^{l}(t)=0
$$

The action value in (10) defines the kernel of a triangular fuzzy interval that represents the supervisory law domain.

- If $P_{l}$ evolves outside the support of $P_{l}^{\text {obj }}$, a fully degraded operating mode is detected (domains $D_{1}$ or $D_{3}$ in Fig. 6). The objective in this case is totally unsatisfied $(\alpha=0)$, and the supervisory action is given by:

$$
\text { If } P_{l} \notin P_{l}^{\mathrm{obj}}(0) \text {, then } r_{s_{i}}^{l}(t)=\Delta-r_{c_{i}}(t)
$$

where

$$
\Delta= \begin{cases}1 & \text {,if } P_{l}<\left(P_{l}^{\mathrm{obj}}\right)^{-}(0) \\ 0 & \text {,if } P_{l}>\left(P_{l}^{\mathrm{obj}}\right)^{+}(0)\end{cases}
$$

In this case, the action consists in either allocating the maximum remaining capacity $(\Delta=1)$ or stop the productivity of the module $(\Delta=0)$. The interval $\left[-r_{c_{i}}, 1-r_{c_{i}}\right]$ defines the support of a triangular fuzzy interval for the supervisory law admissible domain.

- If $P_{l}$ is between the domains $D_{1}$ and $D_{2}$ (Fig. 6) or between $D_{2}$ and $D_{3}$, the system is said in the degraded mode (or switching mode). In these cases, the corresponding $\alpha$-cut of the fuzzy interval $P_{l}^{\text {obj }}$ represents the satisfaction degree. Indeed, when $P_{l}$ is between $D_{1}$ and $D_{2}$, the $\alpha$-cut level is given by the reverse of the left profile function as follows:

$$
\text { If } P_{l} \in\left[\left(P_{l}^{\mathrm{obj}}\right)^{-}(0),\left(P_{l}^{\mathrm{obj}}\right)^{-}(1)\right] \text {, then } \alpha=\left(\left(P_{l}^{\mathrm{obj}}\right)^{-}\left(P_{l}\right)\right)^{-1}=\frac{P_{l}-\left(P_{l}^{\mathrm{obj}}\right)^{-}(0)}{\left(P_{l}^{\mathrm{obj}}\right)^{-}(1)-\left(P_{l}^{\mathrm{obj}}\right)^{-}(0)}
$$

In this case, the supervisory action attempts to allocate the remaining capacity as:

$$
r_{s_{i}}^{l}(t)=(1-\alpha) \cdot\left(1-r_{c_{i}}(t)\right)
$$

On the other hand, when $P_{l}$ is between $D_{2}$ and $D_{3}$, the $\alpha$-cut level is given by the reverse of the right profile function as follows: 


$$
\text { If } P_{l} \in\left[\left(P_{l}^{\mathrm{obj}}\right)^{+}(1),\left(P_{l}^{\mathrm{obj}}\right)^{+}(0)\right] \text {, then } \alpha=\left(\left(P_{l}^{\mathrm{obj}}\right)^{+}\left(P_{l}\right)\right)^{-1}=\frac{P_{l}-\left(P_{l}^{\mathrm{obj}}\right)^{+}(1)}{\left(P_{l}^{\mathrm{obj}}\right)^{+}(0)-\left(P_{l}^{\mathrm{obj}}\right)^{+}(1)}
$$

and the supervisory action attempts to reduce the productivity of the controlled module:

$$
r_{s_{i}}^{l}(t)=(1-\alpha) \cdot\left(-r_{c_{i}}(t)\right)
$$

The actions given by (14) and (16) represent respectively the right and left profiles of a triangular fuzzy interval which represents the supervisory control domain.

The different values of $r_{s_{i}}^{l}(t)$ represent a triangular fuzzy interval denoted by $R_{s_{i}}^{l}$ where its support is $R_{s_{i}}^{l}(0)=\left[-r_{c_{i}}, 1-r_{c_{i}}\right]$ and its kernel is $R_{s_{i}}^{l}(1)=0$. This principle is illustrated in Fig. 8. It can be observed that in the degraded mode, the switching between the operating modes is gradual which may reduce the chattering phenomenon and involve more stable performances.

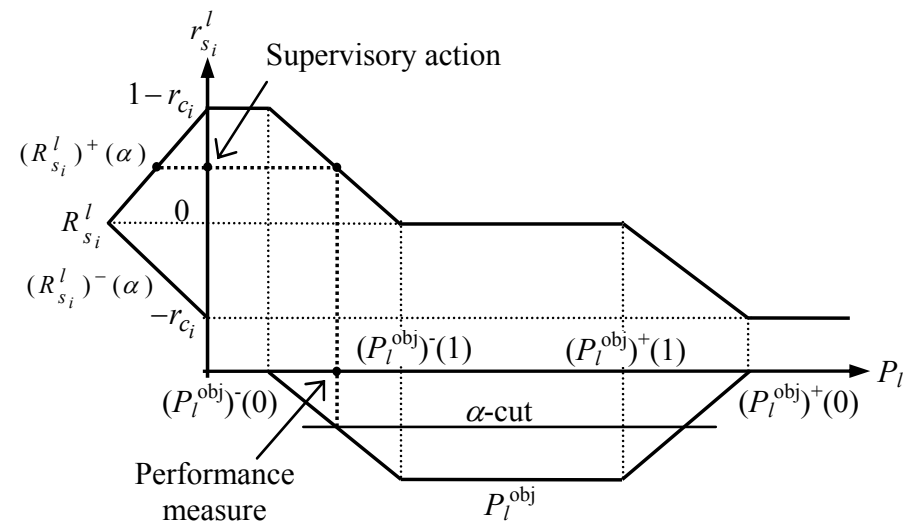

Fig. 8. The evolution of the supervisory control law.

Finally, the different actions $r_{s_{i}}^{l}$ related to each performance indicator $P_{l}$ are aggregated to determine the additive component $r_{s_{i}}$. For example, by exploiting the arithmetic mean operator, the expression of $r_{s_{i}}$ is given by:

$$
r_{s_{i}}(t)=\psi\left(r_{s_{i}}^{1}(t), \ldots, r_{s_{i}}^{L}(t)\right)=\frac{1}{L} \sum_{l=1}^{L} r_{s_{i}}^{l}(t)
$$

The methodology described in this paragraph supposes that the objectives are specified with the same degree of importance. However, in some conflict situations, preferences are attributed to the objectives. For instance, in order to reduce the backlog, one has to increase the system's throughput. Unfortunately, this is achieved by an increased work-in-process (WIP), consequently a trade-off between the WIP and backlog must be found. In these situations, it is more suitable to aggregate (combine) the objectives in a way achieving a best compromise between the multiple conflicting objectives. Thus, the supervisory action results from the evaluation of the aggregated performance measure versus the aggregated objective as described in the following paragraph.

\subsubsection{The aggregation methodology of objectives}

The proposed mechanism here is based on the following three steps:

- The aggregation of the objectives $P_{1}{ }^{\text {obj }}, \ldots, P_{L}{ }^{\text {obj }}$ through an uncertain aggregation operator $\Psi$, since they are defined by fuzzy intervals. This results in a fuzzy aggregated interval $P_{\mathrm{ag}}^{\mathrm{obj}}$.

- The aggregation of the performance measures $P_{1}, \ldots, P_{L}$ by the precise version of the aggregation operator $\Psi$ denoted by $\psi$. This operation leads to an aggregated performance measure $P_{\mathrm{ag}}$.

- The "fuzzification" of the precise aggregated performance measure $P_{\mathrm{ag}}$ on the fuzzy aggregated objective $P_{\mathrm{ag}}^{\mathrm{obj}}$ which results in the satisfaction degree of the overall aggregated performance (the $\alpha$-cut). The supervisory control law is synthesized by following the same principle detailed in the aggregated actions mechanism.

The principle of this methodology is illustrated in Fig. 9. 


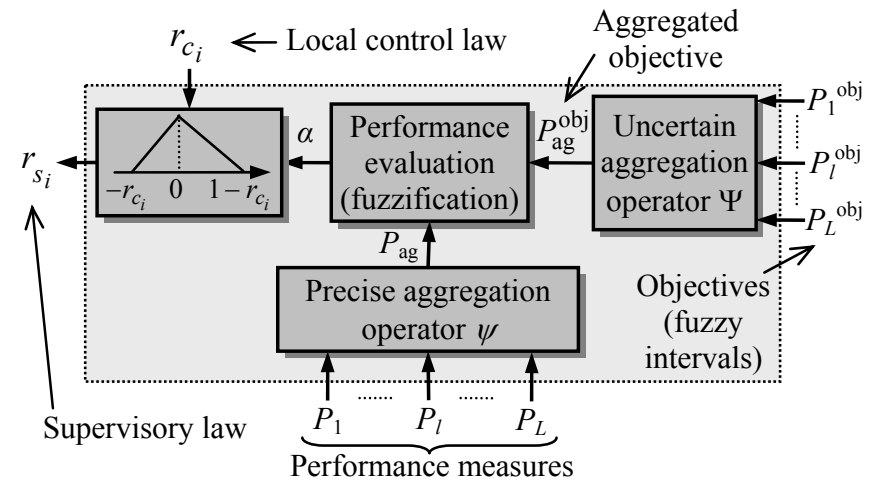

Fig. 9. Synopsis of the objectives aggregation principle.

At the first step, the aggregation of the objectives is to use arithmetic operations on fuzzy intervals. In this case, the arithmetic operations used and defined for conventional intervals can be directly extended to the fuzzy ones according to the profiles representation [9, 21]. In the same way, the $M I N$ and $M A X$ operations for fuzzy intervals can be easily obtained $[10,11,28,29]$. In order to illustrate the aggregation of the objectives, the Choquet integral is used [18, 19,30]. The Choquet integrals are well-known aggregation operators [13, 30] able to handle different types of interactions between the information to be aggregated. In this framework, a particular case of the 2-additive Choquet integral $[18,19]$, where only pairwise interactions are exploited, is considered. Thus, the aggregation of fuzzy objectives by the 2 -additive Choquet integral takes the following form:

$$
\begin{aligned}
& P_{\mathrm{ag}}^{\mathrm{obj}}=\Psi\left[P_{1}^{\mathrm{obj}}, \ldots, P_{L}^{\mathrm{obj}}\right]=\sum_{I_{k l}>0}^{\oplus} M I N\left[P_{k}^{\mathrm{obj}}, P_{l}^{\mathrm{obj}}\right] \cdot I_{k l} \oplus \sum_{I_{k l}<0}^{\oplus} M A X\left[P_{k}^{\mathrm{obj}}, P_{l}^{\mathrm{obj}}\right] \cdot\left|I_{k l}\right| \\
& \oplus \sum_{l=1, \ldots, L}^{\oplus} P_{l}^{\mathrm{obj}}\left(\phi_{l}-\frac{1}{2} \sum_{k \neq l}\left|I_{k l}\right|\right)
\end{aligned}
$$

with respect to the property $\phi_{l}-(1 / 2) \sum_{k \neq l}\left|I_{k l}\right| \geq 0$, and where $\phi_{l}$ are the Shapley indices representing the importance of the objectives. $I_{k l}$ represents the mutual interaction between the performance indicators $P_{k}$ and $P_{l}$. Their values must be in the interval $[-1,1]$ and are interpreted as follows:

- positive $I_{k l}$ means that the performance indicators are complementary,

- negative $I_{k l}$ means that the performance indicators are redundant,

- null $I_{k l}$ means that the performance indicators are independent.

The $M I N$ and the $M A X$ operators are given by:

$$
\left\{\begin{array}{l}
\operatorname{MIN}\left[P_{k}^{\mathrm{obj}}, P_{l}^{\mathrm{obj}}\right]=\left[\min \left(\left(P_{k}^{\mathrm{obj}}\right)^{-}(\alpha),\left(P_{l}^{\mathrm{obj}}\right)^{-}(\alpha)\right), \min \left(\left(P_{k}^{\mathrm{obj}}\right)^{+}(\alpha),\left(P_{l}^{\mathrm{obj}}\right)^{+}(\alpha)\right)\right] \\
\operatorname{MAX}\left[P_{k}^{\mathrm{obj}}, P_{l}^{\mathrm{obj}}\right]=\left[\max \left(\left(P_{k}^{\mathrm{obj}}\right)^{-}(\alpha),\left(P_{l}^{\mathrm{obj}}\right)^{-}(\alpha)\right), \max \left(\left(P_{k}^{\mathrm{obj}}\right)^{+}(\alpha),\left(P_{l}^{\mathrm{obj}}\right)^{+}(\alpha)\right)\right]
\end{array}\right.
$$

The analytical expressions of the $M I N$ and $M A X$ operators are detailed in [10, 28, 29].

The second step of the aggregation mechanism is performed in the same way by considering the performance measures $P_{1}, \ldots, P_{L}$ according to the precise operator $\psi$ :

$$
\begin{aligned}
& P_{\mathrm{ag}}=\psi\left(P_{1}, \ldots, P_{L}\right)=\sum_{I_{k l}>0} \min \left(P_{k}, P_{l}\right) \cdot I_{k l}+\sum_{I_{k l}<0} \max \left(P_{k}, P_{l}\right) \cdot\left|I_{k l}\right| \\
& +\sum_{l=1}^{L} P_{l}\left(\phi_{l}-\frac{1}{2} \sum_{k \neq l}\left|I_{k l}\right|\right)
\end{aligned}
$$

Finally, from the aggregated performance measure $P_{\text {ag }}$ a $\alpha$-cut level is determined on the aggregated fuzzy interval objective $P_{\mathrm{ag}}^{\text {obj }}$. The obtained value is used to determine the additive component $r_{s_{i}}$ (supervisory law) according to the principle given in the paragraph 4.2.1.

\section{Simulation examples}

To illustrate the feasibility of the proposed method, simulations have been carried out through continuous-flow simulator. Two production systems are considered. The first one is a single-part-type transfer line dedicated to 
illustrate the control methodology performance (academic example). The second one is a study case of wafer fabrication in semiconductor industry (the MIT Integrated Circuit Laboratory [2]) used to investigate the applicability of the proposed technique to existent manufacturing systems. The system manufactures multiple types of products with re-entrant flow and involves only transformation operations. The proposed control methodology is implemented by means of Simulink and Floulib toolbox [15] (available at http://www.listic.univsavoie.fr).

\subsection{Illustrative example}

The considered system is composed of four machines with transformation operations and producing one part type. The parameters of machines and the processing times are chosen as follows:

$$
\begin{array}{lll}
\mu_{1}=0.5, & \lambda_{1}=0.3, & \tau_{1}=0.5, \\
\mu_{2}=0.2, & \lambda_{2}=0.05, & \tau_{2}=0.3, \\
\mu_{3}=0.3, & \lambda_{3}=0.2, & \tau_{3}=0.6, \\
\mu_{4}=0.3, & \lambda_{4}=0.01, & \tau_{4}=0.7,
\end{array}
$$

In this case, the feasibility condition (1) of the demand rate is expressed as $d \leq 1$. Thus, giving that the demand rate is 0.7 part per time unit, the parameters of the control module (buffer sizes and hedging point) are listed in Table 2 and computed according to the heuristic given in [2, 3].

Table 2. Control module parameters for $d=0.7$

\begin{tabular}{lllll}
\hline$i$ & $z_{i}^{s}$ & $z_{i}^{b}$ & $x_{i}^{\max }$ & $z_{i}$ \\
\hline 1 & 1.40 & 0.00 & 2 & 3.96 \\
2 & 0.00 & 2.34 & 3 & 2.56 \\
3 & 1.36 & 2.34 & 5 & 2.56 \\
4 & - & - & - & 1.20 \\
\hline
\end{tabular}

The global objectives are specified in terms of backlog and work-in-process minimization. Then, the following performance indicators are adopted:

- The mean end-surplus $\left(P_{1}\right)$,

- The instantaneous end-surplus $\left(P_{2}\right)$,

- The instantaneous work-in-process $\left(P_{3}\right)$.

Both indicators $P_{1}$ and $P_{2}$ are used to keep the production close to the demand (reduce the backlog and the inventory surplus), while $P_{3}$ is used to limit the number of parts in processing (minimize the WIP). The fuzzy interval representations of the associated objectives $P_{l}{ }^{\text {obj }}$ for $l=1, \ldots, 3$; are specified by their supports and kernels as follows:

$$
P_{1}^{\text {obj }}=\left\{\begin{array}{l}
P_{1}^{\mathrm{obj}}(0)=[-3,+3] \\
P_{1}^{\mathrm{obj}}(1)=[-1,+1]
\end{array} ; P_{2}^{\mathrm{obj}}=\left\{\begin{array}{l}
P_{2}^{\mathrm{obj}}(0)=[-3,+3] \\
P_{2}^{\mathrm{obj}}(1)=[-1,+1]
\end{array} ; P_{3}^{\mathrm{obj}}=\left\{\begin{array}{l}
P_{3}^{\mathrm{obj}}(0)=[2,7] \\
P_{3}^{\mathrm{obj}}(1)=[3,6]
\end{array}\right.\right.\right.
$$

Ten simulation runs of 1000 time units for each one have been performed. The evolution of the different performance measures are given by the average over the total ten simulation runs. Fig. 10(a)-(b) shows, in terms of mean values versus time, the evolution of WIP and backlog when applying the following control methods:

- The distributed fuzzy control,

- The supervisory control based on the aggregation of actions using the arithmetic mean operator,

- The supervisory control based on the aggregation of objectives using the 2-additive Choquet integral operator.

Since the performance indicators $P_{1}$ and $P_{2}$ are redundant and both are complement with $P_{3}$, the parameters of the Choquet integral (18) and (20) are chosen according to the expert knowledge as follows: $\phi_{l}=1 / 3$ for $l=1,2$, 3 and $I_{12}=-0.3, I_{13}=0.2, I_{23}=0.2$.

For the tested demand pattern, the analysis of the obtained results leads to the following observations:

- The distributed/supervised approaches achieve a substantial reduction of backlog and relative improvement of WIP compared with the distributed fuzzy control.

- The distributed/supervised control based on the aggregation of objectives reaches better performances when compared with the method based on the aggregation of actions.

- When using the 2-additive Choquet integral operator, backlog and WIP are maintained in their admissible domain bounds. 


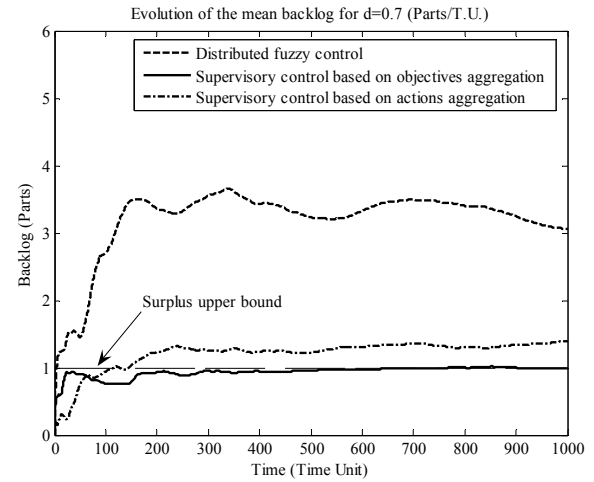

(a)

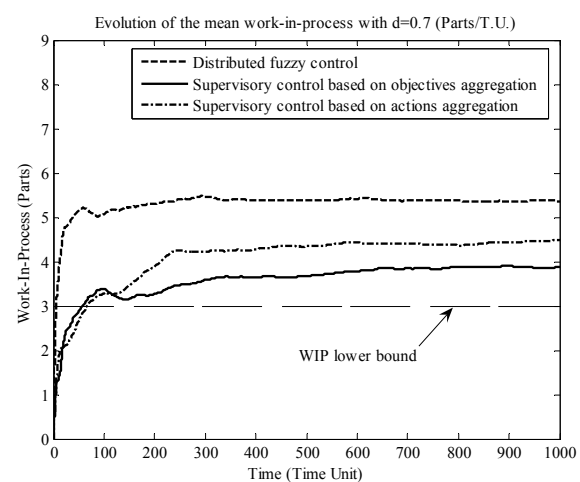

(b)

Fig. 10. Control performances: (a) Backlog, (b) WIP.

The performance of the control methodology is highly dependent on the demand levels and patterns. Consequently, a constant demand rate close to the maximum productivity of the system is considered. In this case, when considering the WIP and backlog as performance measures, the supervisory control leads to reduce the backlog since it is more important (in the action law) than the WIP. However, when the demand rate is low, the backlog is at low levels since there is enough capacity to satisfy the demand. In this case, the distributed and supervised approaches may achieve comparable performances. These results are due to the fact that the WIP and backlog are conflicting performance measures and a best compromise must be achieved between them.

\subsection{Application to the multiple-part-type systems}

As the complexity of a production system is related to its dimension, uncertainties, variety of products, re-entrant sequences, etc., an application to actual multiple-part-type processes is studied in this paragraph [2, 20, 42].

\subsubsection{Multipe-part-type formulation}

Let consider a single production module $P M(i)$ that operates on different part types $j$ such that $j \in Q(i)$, where $Q(i)$ the set of part types processed on $P M(i)$, each of them may involves $K_{i j}\left(k=1, \ldots, K_{i j}\right)$ different operations. In this case, the original module $P M(i)$ is virtually divided into $\sum_{\left\{j \mid j \in Q_{i}\right\}} K_{i j}$ single-part-type sub-modules. Thus, there are as many sub-modules as the number of the operations to be performed in the original module $P M(i)$.

Let us denote $p m(i, j, k)$ be the sub-module of $P M(i)$ which performs the $k$ th operation on the part of type $j$ and $\tau_{i j k}$ its processing time. When considering a constant demand rate $d_{j}$ of the production of the part type $j$, the feasibility condition becomes:

$$
\sum_{\{j \mid j \in Q(i)\}} \sum_{k=1}^{K_{i j}} d_{j} \tau_{i j k} \leq e_{i}, i=1, \ldots, N
$$

To distribute the machine operating time to the different part types, and consequently the decision of the production rate $u_{i j k}$ of every sub-module $p m(i, j, k)$, the assumption that all part types are of equal importance is considered. In this case, the maximum production rate of the sub-module $p m(i, j, k)$ is equal to:

$$
u_{i j k}^{\max }=A_{i j k} \cdot\left(\frac{1}{\tau_{i j k}}\right) \text {, with } A_{i j k}=\left(\frac{d_{j} \tau_{i j k}}{\sum_{\{j \mid j \in Q(i)\}} \sum_{k=1}^{K_{i j}} d_{j} \tau_{i j k}}\right)
$$

And the production rate $u_{i j k}$ of each operation $k$ of every product $j$ would be:

$$
u_{i j k}(t)=r_{i j k}(t) u_{i j k}^{\max }=\left(r_{c i j k}(t)+r_{s_{j j k}}(t)\right) \cdot u_{i j k}^{\max }
$$

where $r_{c_{i j k}}$ is the outcome of the local fuzzy sub-control module associated to the sub-module $p m(i, j, k)$, and $r_{s_{i j k}}$ the additive control component provided by the supervisor of the flow of part type $j$. The remaining operating time of $P M(i)$ is calculated as follows:

$$
R T_{i}(t)=1-\sum_{\{j \mid j \in Q(i)\}} \sum_{k=1}^{K_{i j}} r_{i j k}(t) A_{i j k}
$$


The value of (24) is distributed to the operations with the highest priority. It is given to the operations having the global control law $r_{i j k}$ equal to 1 . The extra machine time devoted to the operations with the highest priority is:

$$
E_{i j k}(t)=R T_{i}(t) \cdot C_{i j k}(t)
$$

where

$$
C_{i j k}(t)=\left(\frac{d_{j} \tau_{i j k} \beta_{i j k}(t)}{\sum_{\{j \mid j \in Q(i)\}} \sum_{k=1}^{K_{i j}} d_{j} \tau_{i j k} \beta_{i j k}(t)}\right) \text {, and } \beta_{i j k}(t)= \begin{cases}0 & \text { if } r_{i j k}(t)<1 \\ 1 & \text { if } r_{i j k}(t)=1\end{cases}
$$

Finally, the production rate of the sub-module $p m(i, j, k)$ is:

$$
u_{i j k}(t)=r_{i j k}(t) u_{i j k}^{\max }+E_{i j k}(t) \cdot\left(\frac{1}{\tau_{i j k}}\right)
$$

\subsubsection{Semiconductor application}

In order to illustrate the feasibility of the supervisory control approaches in the case of multiple-part-type systems with re-entrant flow, an application of two actual processes of wafer fabrication in semiconductor industry is studied [2]. The first process called poly-gate capacitor consists of 17 operations and the second called poly-monitor involves 7 operations.

The part type 1 follows the poly-gate capacitor process while the type 2 goes through the system following the poly-monitor process. The parameters of machines are listed in Table 3 . The processing times are in Table 4 where the time unit is hour and the unit of part is lot.

Table 3. Machine parameters (1/hour).

\begin{tabular}{|c|c|c|c|c|c|c|c|c|c|c|c|}
\hline \multirow[t]{2}{*}{ Process $(j)$} & \multirow[t]{2}{*}{ Operation $(k)$} & \multicolumn{10}{|c|}{ Machine $(i)$} \\
\hline & & 1 & 2 & 3 & 4 & 5 & 6 & 7 & 8 & 9 & 10 \\
\hline \multirow{3}{*}{$\begin{array}{l}\text { Process 1: } d_{1}= \\
0.6 \text { (lot/hour) }\end{array}$} & 1 & 0.25 & 0.469 & 0.969 & 0.469 & 0.469 & 0.25 & 0.156 & 0.188 & 0.131 & 0.469 \\
\hline & 2 & 0.25 & - & - & - & - & - & 0.156 & 0.188 & 0.131 & 0.469 \\
\hline & 3 & - & - & - & - & - & - & - & 0.188 & 0.163 & - \\
\hline $\begin{array}{l}\text { Process 2: } d_{2}= \\
0.5 \text { (lot/hour) }\end{array}$ & 1 & 0.25 & - & 0.656 & 0.469 & 0.469 & - & 0.156 & - & 0.163 & 0.469 \\
\hline
\end{tabular}

\begin{tabular}{lllllllllll}
\hline & \multicolumn{1}{l}{ Machine $(i)$} & & & & & & & & \\
\cline { 2 - 11 } & 1 & 2 & 3 & 4 & 5 & 6 & 7 & 8 & 9 & 10 \\
\hline$\lambda_{i}$ & 0.01 & 0.001 & 0.002 & 0.002 & 0.017 & 0.002 & 0.002 & 0.01 & 0.001 & 0.01 \\
\hline$\mu_{i}$ & 0.5 & 0.5 & 0.5 & 0.5 & 0.33 & 0.5 & 0.33 & 0.5 & 0.5 & 0.33 \\
\hline
\end{tabular}

Table 4. The processing times $\tau_{i j k}$ for the two processes (hours).

The feasibility condition (1) provides $d_{1} \leq 0.65$ and $d_{2} \leq 0.54$. Giving the demand rates of 0.6 (lot/hour) for the poly-gate capacitor process and 0.5 (lot/hour) for the poly-monitor process, the parameters of each sub-control module (buffer sizes and hedging levels) are calculated and respectively listed in Table 5 and Table 6.

Table 5. The parameters of the sub-controllers for the process 1 .

\begin{tabular}{llllllllllll}
\hline \multicolumn{7}{c}{ Machine $(i)$} \\
\cline { 2 - 12 } & & 1 & 2 & 3 & 4 & 5 & 6 & 7 & 8 & 9 & 10 \\
\hline Surplus & $z_{i, 1,1}$ & 6.307 & 3.907 & 6.307 & 2.088 & 5.107 & 0.888 & 3.907 & 3.907 & 5.107 & 5.107 \\
hedging & $z_{i, 1,2}$ & 5.107 & - & - & - & - & - & 0.888 & 2.088 & 2.088 & 0.888 \\
levels & $z_{i, 1,3}{ }^{s}$ & - & - & - & - & - & - & & 0.888 & 0.888 & 0 \\
\hline Starvation & $z_{i, 1,1}{ }^{s}$ & 0 & 0 & 1.2 & 1.2 & 0 & - & 1.818 & 0 & 0 & 0 \\
parameters & $z_{i, 1,2}{ }^{s}$ & 1.2 & - & - & - & - & - & 0 & 0 & 0 & 0 \\
& $z_{i, 1,3}{ }^{2}$ & - & - & - & - & - & - & - & 0 & 0 & - \\
\hline Buffer sizes & $x_{i, 1,1}{ }^{\max }$ & 1 & 1 & 5 & 3 & 1 & - & 2 & 1 & 4 & 2 \\
& $x_{i, 1,2}{ }^{\max }$ & 1 & - & - & - & - & - & 2 & 1 & 1 & 2 \\
& $x_{i, 1,3}{ }^{\max }$ & - & - & - & - & - & - & - & 2 & 2 & - \\
\hline
\end{tabular}


Table 6. The parameters of the sub-controllers for the process 2.

\begin{tabular}{|c|c|c|c|c|c|c|c|c|c|c|}
\hline & \multicolumn{10}{|c|}{ Machine $(i)$} \\
\hline & 1 & 2 & 3 & 4 & 5 & 6 & 7 & 8 & 9 & 10 \\
\hline$\overline{z_{i, 2,1}}$ & 4.315 & - & 3.115 & 1.915 & 1.915 & - & 0.715 & - & 0.715 & 0.715 \\
\hline$\overline{z_{i, 2,1}{ }^{s}}$ & 1.2 & - & 1.2 & 1.2 & 0 & - & - & - & 0 & 0 \\
\hline$x_{i, 2,1}^{\max }$ & 1 & - & 5 & 1 & 1 & - & - & - & 2 & 2 \\
\hline
\end{tabular}

The performance measures used in the supervisory mechanism for each process $(j=1,2)$ are: the instantaneous end-surplus $\left(P_{j 1}\right)$, the work-in-process $\left(P_{j 2}\right)$ and the production lead time $\left(P_{j 3}\right)$. The objectives are expressed in terms of mean value for the backlog, the work-in-process and the lead time for each part type. In order to establish the tolerance intervals related to each objective, one has to estimate the limit bounds of the desired performance measures. For the surplus, the tolerance interval is chosen in such a way to keep the production close to the demand: $P_{j 1}{ }^{\text {obj }}=[-1,1]$. The overall WIP level in the system is the sum of all parts in buffers and parts processed on machines. Since the buffer sizes are calculated with regard to the starvation parameters $z_{i, j, k}^{s}$, the $\mathrm{WIP}_{j}$ bounds are approximated by:

$$
\sum_{\{i \mid j \in Q(i)\}} \sum_{k=1}^{K_{i j}} z_{i, j, k}^{s} \leq \mathrm{WIP}_{j}(t) \leq \sum_{\{i \mid j \in Q(i)\}} \sum_{k=1}^{K_{i j}} x_{i, j, k}^{\max }, j=1,2
$$

The real values of buffer sizes and starvation parameters are approximated to integer values. Referring to the values in Table 5 and Table 6, equation (28) leads to: $5 \leq \mathrm{WIP}_{1}(t) \leq 31$ and $3 \leq \mathrm{WIP}_{2}(t) \leq 12$. Thus, the related tolerance intervals are chosen as follows: $P_{12}{ }^{\text {obj }}=[5,10] ; P_{22}{ }^{\text {obj }}=[3,6]$. Based on these values and using the Little's law $\left(W I P_{j}=d_{j} T_{j}\right)$, the tolerance intervals for the production lead time are fixed as: $P_{13}{ }^{\text {obj }}=[9,16] ; P_{23}{ }^{\text {obj }}=$ $[6,12]$.

The supervision based on the objectives aggregation is adopted with the 2-additive Choquet integral operator. This choice is motivated by the fact that the performances of the backlog and the WIP are conflicting. Since the WIP and the lead time express the same aspect of performance, they exhibit redundant aspect. Thus, according to experts, the associated Choquet integral parameters are: $\phi_{j l}=1 / 3$ for $l=1,2,3$ and $I_{12}=0.2, I_{13}=0.25, I_{23}=-0.3$, the others being equal to 0. Comparative results for the backlog, WIP and production lead time for both processes are respectively shown in Fig. 11, Fig. 12 and Fig. 13.
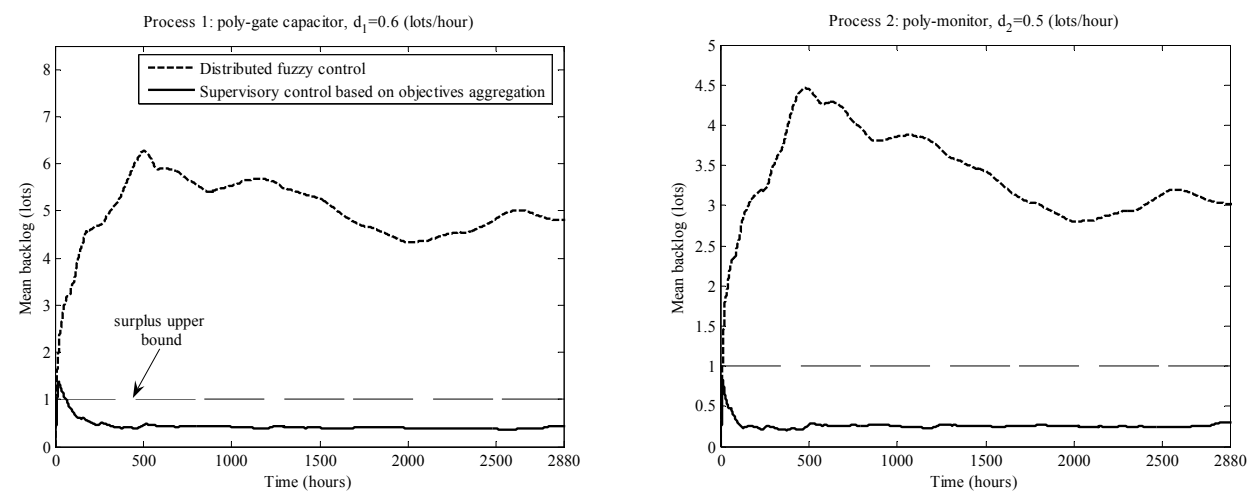

Fig. 11. Mean backlog: (a) part type 1, (b) part type 2 .
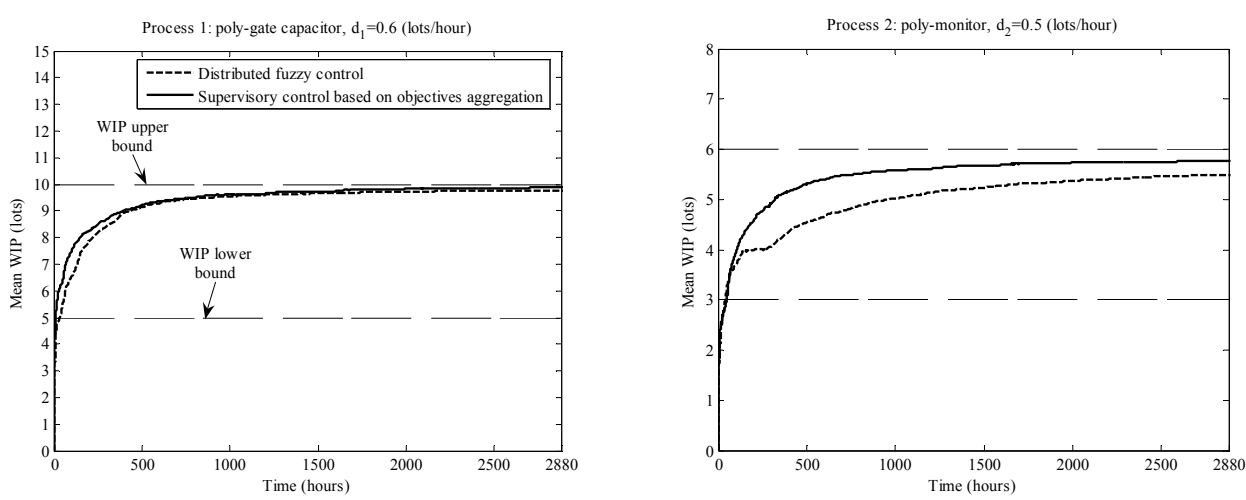

Fig. 12. Mean WIP: (a) part type 1, (b) part type 2. 

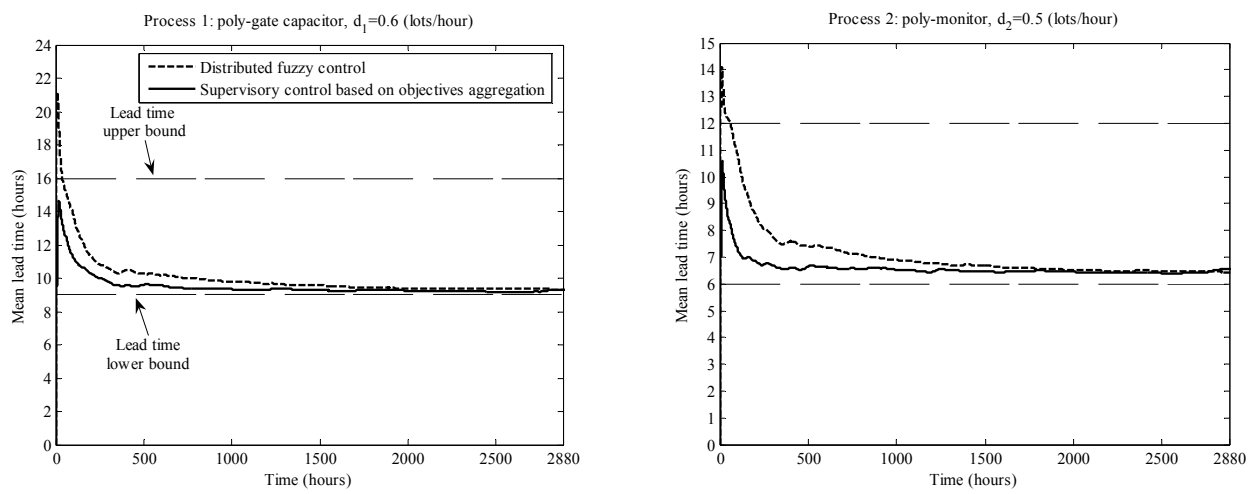

Fig. 13. Mean lead time: (a) part type 1, (b) part type 2.

Table 7 shows the statistical significance of the results obtained in 10 simulation runs. Each run represents about 12 months of production (2880 hours).

Based on the obtained results, the distributed/supervised control approach achieves a substantial reduction of backlog for both part types (Fig. 11). The performances of the WIP and lead time for each part type are similar in both methods but they are maintained within their tolerance intervals (Fig. 12, Fig. 13). These results are due to the fact that the supervisory mechanism provides an action in such a way that some trade-off is achieved with regard to the objective values, their importance and interactions. Indeed, as the performances of the distributed control in terms of WIP and lead time are satisfied, the supervisor provides an action which allocates the remaining capacity in order to reduce the backlog. In this case, the Choquet integral operator ensures the coherence of the satisfaction degrees of each performance by taking into account the relative importance of the performances and their mutual interactions.

We can conclude that the distributed/supervised control strategy exhibits better performance in almost every case. These results are very promising, since the decision method is very flexible and combining several control objectives with the local control one. This may help to cope with the multiple and conflicting control objectives and make the decision-making process more efficient in the production-flow control.

Table 7. Statistical significance of the simulation results.

\begin{tabular}{|c|c|c|c|c|c|c|c|}
\hline & & \multicolumn{3}{|c|}{ Distributed fuzzy control } & \multicolumn{3}{|c|}{$\begin{array}{l}\text { Supervisory control based on } \\
\text { objectives aggregation }\end{array}$} \\
\hline & & $\begin{array}{l}\text { WIP } \\
\text { (lots) }\end{array}$ & $\begin{array}{l}\text { Backlog } \\
\text { (lots) }\end{array}$ & $\begin{array}{l}\text { Lead time } \\
\text { (hours) }\end{array}$ & $\begin{array}{l}\text { WIP } \\
\text { (lots) }\end{array}$ & $\begin{array}{l}\text { Backlog } \\
\text { (lots) }\end{array}$ & $\begin{array}{l}\text { Lead time } \\
\text { (hours) }\end{array}$ \\
\hline \multirow{4}{*}{$\begin{array}{l}\text { Process 1: } d_{1}=0.6 \\
\text { (lots/hour) }\end{array}$} & Mean & 9.768 & 4.801 & 9.300 & 9.908 & 0.447 & 9.316 \\
\hline & $\begin{array}{l}\text { Standard } \\
\text { deviation }\end{array}$ & 0.136 & 1.505 & 0.199 & 0.128 & 0.167 & 0.303 \\
\hline & Maximum & 9.883 & 7.059 & 9.567 & 10.052 & 0.642 & 9.745 \\
\hline & $\begin{array}{l}\text { Confidence } \\
\text { level }\end{array}$ & 0.99 & 0.95 & 0.99 & 0.99 & 0.99 & 0.99 \\
\hline \multirow{4}{*}{$\begin{array}{l}\text { Process 2: } d_{2}=0.5 \\
\text { (lots/hour) }\end{array}$} & Mean & 5.491 & 3.026 & 6.433 & 5.771 & 0.296 & 6.541 \\
\hline & $\begin{array}{l}\text { Standard } \\
\text { deviation }\end{array}$ & 0.063 & 0.943 & 0.187 & 0.065 & 0.128 & 0.336 \\
\hline & Maximum & 5.590 & 4.392 & 6.594 & 5.875 & 0.450 & 6.883 \\
\hline & $\begin{array}{l}\text { Confidence } \\
\text { level }\end{array}$ & 0.95 & 0.99 & 0.975 & 0.95 & 0.99 & 0.99 \\
\hline
\end{tabular}

\section{Conclusion}

In this article, intelligent distributed and supervised control architecture for continuous-flow production systems has been presented. The hierarchical structure consists of a lower level of distributed fuzzy controllers, which is supervised by a higher level of decision-making. The lower level regulates the production flow by adjusting the machine processing rates. It uses a fuzzy controller based on the Takagi-Sugeno fuzzy system. The higher level of supervision monitors the system by using global performance indicators. The supervisory mechanism is based on aggregation operator mechanisms which provide an additive component to the local controller when degraded operating modes are detected. The supervisor is built according to the satisfaction degree of the different and possibility conflicting objectives quantified by fuzzy intervals.

For the studied cases, the obtained results show a promise improvement of performances when compared with the unsupervised distributed control. To summarize, the main advantages of the proposed control approach are: 
- It takes into account the different degrees of importance for each control objective,

- It facilitates the implementation phase due to the modularity and the distributivity of the control architecture.

The robustness of the proposed approach is evaluated regardless to the demand variation (random variation) in [44]. The obtained results show acceptable improvement of performances providing that the demand changes don't exceed the system's capacity. An attractive extension is to study the structured methods traditionally used in the multiple criteria decision problems in order to quantify the fuzzy intervals of the desired objectives.

\section{References}

[1] Angsana A. and Passino K.M., "Distributed Fuzzy Control of Flexible Manufacturing Systems", IEEE Transactions on Control Systems Technology, Vol. 2, N 4, pp. 423-435, 1994.

[2] Bai X., "Scheduling Manufacturing Systems with Work-In-Process Inventory Control", Ph. D. thesis, Operations Research Center, MIT, Cambridge, 1991.

[3] Bai X. and Gershwin S.B., "Scheduling Manufacturing Systems with Work-In-Process Inventory Control: Single-PartType Systems", IIE Transactions, Vol. 27, pp. 599-617, 1995.

[4] Balduzzi F. and Menga G., "A State Variable Model for the Fluid Approximation of Flexible Manufacturing Systems", In Proceeding of the IEEE International Conference on Robotics \& Automation, Leuven, Belgium, pp. 1172-1178, 1998.

[5] Brandimarte P., Sharifnia A. and Von Turkovich B.F., "Continuous Flow Models of Manufacturing Systems: a Review", CIRP Annals - Manufacturing Technology, Vol. 45, N 1, pp. 441-444, 1996.

[6] Bongaerts L., Monostori L., McFarlane D. and Kádár B., "Hierarchy in distributed shop floor control", Computers in Industry, Vol. 43, $\mathrm{N}^{\circ}$ 2, pp. 123-137, 2000.

[7] Boukezzoula R., Galichet S., Foulloy L., "Nonlinear Internal Model Control: Application of Inverse Model Based Fuzzy Control”, IEEE Transactions on Fuzzy Systems, Vol. 11, Nº 6, pp. 814-829, 2003.

[8] Boukezzoula R., Foulloy L. and Galichet S., "Inverse Controller Design for Interval Fuzzy Systems", IEEE Transactions On Fuzzy Systems, Vol. 14, N 1, pp. 111-124, February, 2006.

[9] Boukezzoula R., Galichet S. and Foulloy L., "Inverse arithmetic operators for fuzzy intervals", In Proceeding of $5^{\text {th }}$ Conference of the European Society for Fuzzy Logic and Technology (EUSFLAT'07), Ostrava, République Tchèque, Vol. 2, pp. 279-286, September 2007.

[10] Boukezzoula R., Galichet S., Foulloy L., "MIN and MAX Operators for Fuzzy Intervals and their Potential Use in Aggregation Operators”, IEEE Transactions on Fuzzy Systems, Vol. 15, № 6, pp. 1135-1144, December 2007.

[11] Chiu C.-H. and Wang W.-J., “A simple computation of MIN and MAX operations for fuzzy numbers”, Fuzzy Sets and Systems, Vol. 126, N², pp. 273-276, 2002.

[12] Custodio L.M.M., Sentieiro J.J.S. and Bispo C.F.G., "Production planning and scheduling using a fuzzy decision system", IEEE Transactions on Robotics and Automation, Vol. 10, № 2, pp.160-168, 1994.

[13] Dubois D. and Prade H., "On the use of aggregation operations in information fusion processes”, Fuzzy Sets Systems, Vol. 142, $\mathrm{N}^{\circ}$ 1, pp. 143-161, 2004.

[14] Duffie N.A. and Prabhu V.V., "Heterarchical control of highly distributed manufacturing systems", International Journal of Computer Integrated Manufacturing, Vol. 13, N², pp. 270-281, 1996.

[15] Foulloy L., Boukezzoula R. and Galichet S., "An educational tools for fuzzy control”, IEEE Transactions on Fuzzy Systems, Vol. 14, N², pp.217-221, 2006.

[16] Galichet S., Boukezzoula R. and Foulloy L., "Explicit analytical formulation and exact inversion of decomposable fuzzy systems with singleton consequents", Fuzzy Sets and Systems, Vol. 146, N 3, pp. 421-436, 2004.

[17] Gershwin S.B., "Design and Operation of Manufacturing Systems - The Control Point Policy", IIE Transactions, Vol. 32, No 2, pp.891-906, 2000.

[18] Grabisch M. and Roubens M., "Application of the Choquet integral in multicriteria decision making”, In Fuzzy Measure and Integrals, New York: Physica-Verlag, pp. 348-374, 2000.

[19] Grabisch M. et Perny P., "Logique floue, principes, aide à la décision : chapitre Agrégation multicritère", Traité IC2, série informatique et systèmes d'information, Paris Hermès Science Publications, 2003.

[20] Ioannidis S., Tsourveloudis N.C. and Valavanis K., "Fuzzy supervisory control of manufacturing systems", IEEE Transactions on Robotics and Automation, Vol. 20, N 3, pp.379-389, 2004.

[21] Klir G.J., "Fuzzy arithmetic with requisite constraints," Fuzzy Sets and Systems, Vol. 91, º 2, pp. 165-175, 1997.

[22] Kotak D., Wu S., Fleetwood M. and Tamoto H., "Agent-based holonic design and operations environment for distributed manufacturing”, Computers in Industry, Vol. 52, N² 2, pp. 95-108, 2003. 
[23] Koestler A., "The Ghost in the Machine”, Arkana Books, London, 1969.

[24] Kouikoglou V.S. and Phillis Y.A., "A continuous-flow model for production networks with finite buffers, unreliable machines, and multiple products", International Journal of Production Research, Vol. 35, pp.381-397, 1997.

[25] Lee Y.F., Jiang Z.B. and Liu H.R., "Multiple-objective scheduling and real-time dispatching for the semiconductor manufacturing system", Computers \& Operations Research, 2007.

[26] Leitão P. and Restivo F., "A holonic approach to dynamic manufacturing scheduling", Robotics and ComputerIntegrated Manufacturing, 2007.

[27] Mamdani E.H., "Application of Fuzzy Algorithms for Control of a simple Dynamic Plant", In proceeding of the IEE Control and Science, Vol. 121, N 12, pp. 1585-1588, 1974.

[28] Megri F. and Boukezzoula R., "MIN and MAX Operators for Trapezoidal Fuzzy Intervals. Part I: Formalization and Application", Fuzzy-IEEE Conference, Hong Kong, 2008.

[29] Megri F. and Boukezzoula R., "MIN and MAX Operators for Trapezoidal Fuzzy Intervals. Part II: Analytical Expressions Proof”, Fuzzy-IEEE Conference, Hong Kong, 2008.

[30] Meyer P. and Roubens M., "On the use of the Choquet integral with fuzzy numbers in multiple criteria decision support," Fuzzy Sets and Systems, Vol. 157, N 7, pp. 927-938, 2006.

[31] Passino K.M., and Yurkovish S., "Fuzzy Control”, CA: Addison-Wesley Longman, Menlo Park, 1998.

[32] Perkins J., and Kumar P.R., "Stable Distributed Real-Time Scheduling of Flexible Manufacturing / Assembly / Disassembly Systems”, IEEE Transactions on Automatic Control, Vol. 34, № 2, pp.139-148, 1989.

[33] Rovithakis G.A., Gaganis V.I., Perrakis S.E. and Christodoulou M.A., "Real-time control of manufacturing cells using dynamic neural network", Automatica, Vol. 35, № 1, pp. 139-149, 1999.

[34] Samanta B. and Al-Araimi S.A., "An inventory control model using fuzzy logic”, International Journal of Production Economics, Vol. 73, Nº 3, pp. 217-226, 2001.

[35] Sharifnia A., "Stability and Performance of Distributed Production Control Methods based on Continuous-Flow Models", IEEE Transactions on Automatic Control, Vol. 39, N 4, pp. 725-737, 1994.

[36] Shen W., Norrie D.H., “Agent-based systems for intelligent manufacturing: a state-of-the-art survey”, Knowledge and Information Systems: an International Journal, Vol. 1, N², pp. 129-156, 1999.

[37] Shen W., Hao Q. Yoon H.J. and Horrie D.H., "Applications of agent-based systems in intelligent manufacturing: an update review”, Advanced Engineering Informatics, Vol. 20, 2006.

[38] Suhail A. and Khan Z.A., "Fuzzy control with limited control opportunities and response delay-a production-inventory control scenario", International Journal of Approximate Reasoning, Vol. 38, N 1, pp.113-131, 2005.

[39] Sugeno M., "On stability of fuzzy systems expressed by fuzzy rules with singleton consequents", IEEE Transactions on Fuzzy set and Systems, Vol. 7, N², pp. 201-224, 1999.

[40] Takagi T. and Sugeno M., "Fuzzy identification of systems and its applications to modelling and control", IEEE Transactions on Systems, Man and Cybernetics, Vol. 15, pp. 116-132, 1985.

[41] Tamani K., Habchi G., Boukezzoula R., "Fuzzy Supervision of Adaptive Fuzzy Controllers for Manufacturing Systems", IEEE Int. Conf. on Computational Intelligence for Modelling, Control and Automation (CIMCA 2006), Sydney, Australia, November 2006.

[42] Tamani K., Boukezzoula R, Habchi G., "Hierarchical control for continuous flow simulation of manufacturing systems", Proceedings of the $6^{\text {th }}$ EUROSIM Congress on Modelling and Simulation, Ljubljana, Slovenia, September, 2007.

[43] Tamani K., Boukezzoula R., Habchi G., "Supervisory based capacity allocation control for manufacturing systems simulation", Accepted for publication in International Journal of Manufacturing Technology and Management, 2009.

[44] Tamani K., Boukezzoula R., Habchi G., "Multiple-Objective Supervisory Control based on the Aggregation Operators in a Production System with Frequent Demand Change", Submitted to the $13^{\text {th }}$ IFAC Symposium on Information Control Problems in Manufacturing (INCOM 2009), Moscow, Russia, June 2009.

[45] Trentesaux D., "Pilotage hétérarchique des systèmes de production", Habilitation à Diriger des Recherches, Université de Valenciennes et du Hainaut-Cambrésis, France, 2002.

[46] Tsourveloudis N.C., Dretoulakis E. and Ioannidis S., "Fuzzy work-in-process inventory control of unreliable manufacturing systems", Information Sciences, Vol. 127, pp. 69-83, 2000.

[47] Tsourveloudis N.C., Doitsidis L., Ioannidis S., "Work-in-process scheduling by evolutionary tuned fuzzy controllers", International Journal of Advanced Manufacturing Technology, Vol. 34, N 7-8, pp. 748-761, 2007.

[48] Vargas-Villamil F.D., Rivera D.E., Kempf K.G., "A hierarchical approach to production control of reentrant semiconductor manufacturing lines", IEEE Transactions On Control Systems Technology, Vol. 11, N 4, pp. 578-587, 2003. 